\title{
BMJ Open Prognostic factor analysis in patients with temporomandibular disorders after reversible treatment: study protocol for a prospective cohort study in China
}

\author{
Ling Zhang, ${ }^{1}$ Wentao Shi, ${ }^{2}$ Shenji Lu, ${ }^{1}$ Bin Cai ${ }^{10},{ }^{1}$ Shuai Fan, ${ }^{1}$ Yang Yang, ${ }^{1}$ Lili Xu ${ }^{1}$
}

To cite: Zhang L, Shi W, Lu S, et al. Prognostic factor analysis in patients with temporomandibular disorders after reversible treatment: study protocol for a prospective cohort study in China. BMJ Open 2021;11:e048011. doi:10.1136/ bmjopen-2020-048011

- Prepublication history for this paper is available online. To view these files, please visit the journal online (http://dx.doi. org/10.1136/bmjopen-2020048011).

Received 15 December 2020 Accepted 09 June 2021

Check for updates

(c) Author(s) (or their employer(s)) 2021. Re-use permitted under CC BY-NC. No commercial re-use. See rights and permissions. Published by BMJ.

${ }^{1}$ Department of Rehabilitation, Shanghai Jiao Tong University School of Medicine Affiliated Ninth People's Hospital,

Shanghai, China

${ }^{2}$ Clinical Research Center,

Shanghai Jiao Tong University School of Medicine Affiliated Ninth People's Hospital,

Shanghai, China

Correspondence to

Dr Lili Xu; hebll|@163.com

\section{ABSTRACT}

Introduction Temporomandibular disorders (TMDs) are complex multifactorial disorders. Reversible treatment has been suggested for the initial management of TMD; however, comparable therapeutic effects of different reversible intervention modalities remain controversial. Various biopsychosocial factors, which may be putative prognostic factors that influence the response to reversible treatment for TMD, have been reported to increase the risk of developing first-onset TMD. However, there is a paucity of research that aims to identify prognostic factors associated with the clinical outcomes of reversible treatment in people with TMD. The objective of this prospective cohort study is to identify prognostic factors that are associated with clinical outcomes of reversible treatment in patients with TMD and to analyse the risk factors that influence the development of chronic TMD. Methods and analysis We plan to recruit 834 patients with TMD who meet the inclusion criteria. Once informed consent is obtained, baseline data, including anamnestic data, physical assessments and self-report questionnaires, will be collected from participants at their first clinic visit; subsequently, they will receive 1-4 weeks of reversible treatment. The primary treatment outcome measures will be a change in the anterior maximum mouth opening, worsening of TMD pain scores assessed using a visual analogue scale (VAS) and a reduction in characteristic pain intensity. A good outcome will be defined as an anterior maximal opening $\geq 35 \mathrm{~mm}$ and at least a $30 \%$ reduction in VAS scores 3 months after baseline. The association between candidate prognostic factors and clinical outcomes of reversible TMD treatment will be analysed. Ethics and dissemination The protocol has been approved by the Ethics Committee of Ninth People's Hospital, Shanghai Jiao Tong University School of Medicine, Shanghai, China, based on the guidelines outlined in the Declaration of Helsinki (SH9H-2019-T316-4). The results of this study will be reported in accordance with the Strengthening the Reporting of Observational Studies in Epidemiology statement. The authors intend to publish the results in a peer-reviewed journal.

Trial registration number ChiCTR2000033328.

\section{INTRODUCTION}

Temporomandibular disorders (TMDs) are painful musculoskeletal conditions that are
Strengths and limitations of this study

- A battery of assessments of demographic characteristics and biopsychosocial factors will be conducted to evaluate the associations between those variables and the prognosis of patients with temporomandibular disorder (TMD).

- Putative risk factors that are associated with the development of chronic TMD will be explored so that the heterogeneity of patient characteristics and psychosocial factors may be considered during treatment planning.

- Anterior maximal opening of the mouth and TMD pain will be included as outcome measures, providing evidence that will be useful for appropriately managing Chinese patients with TMD.

- As putative prognostic factors are selected based on current knowledge of risk factors associated with the development of TMD, some possible biopsychosocial factors may not be assessed in this study.

- Considering that the ethnic background of TMD patients may be associated with psychological variables, the influence of these psychological factors on the prognosis of patients with TMD may also differ based on ethnicity.

associated with pain and dysfunction of the temporomandibular joint (TMJ) and masticatory muscles. ${ }^{12}$ Approximately $5 \%-12 \%$ of adults experience TMD. ${ }^{2}$ The most common TMD symptoms and signs are facial pain, impaired jaw mobility, deviations of mandibular movements and TMJ sounds, affecting the patient's well-being and quality of life. ${ }^{3}$

TMD is a complex disorder associated with multiple physical, psychological, genetic, sensory processing and environmental domains, and clinical characteristics have been identified to predict the increased risk of developing TMD. ${ }^{4}$ Previous reports from the Orofacial Pain: Prospective Evaluation and Risk Assessment (OPPERA) study have concluded that numerous biopsychosocial factors increase the risk of developing 
first-onset TMD. ${ }^{4-6}$ The OPPERA study findings demonstrated that a complex pattern of considerable changes in psychological functioning (ie, perceived stress, depression and somatisation), ${ }^{7}$ pain sensitivity, ${ }^{8}$ clinical jaw function, ${ }^{9}$ sleep disturbance and other health conditions ${ }^{10}$ is associated with changes in the TMD status.

Various interventions have been suggested for TMD; although to date, the most effective treatment programme remains unclear. ${ }^{11-14}$ These interventions are grouped into three categories based on the level of invasiveness, including reversible (eg, education, self-management, splint therapy and physiotherapy), minimally invasive (eg, arthrocentesis) and surgical (eg, arthroscopic and open joint surgeries) interventions. ${ }^{11}$ A similar painreducing effect of physiotherapy and splint therapy has been confirmed in patients with myofascial TMD pain. ${ }^{14}$ Diraçoğlu $e t a l^{13}$ concluded that neither reversible methods nor arthrocentesis was beneficial in the early treatment of TMD. A previous randomised controlled trial found no difference between medical management, nonsurgical rehabilitation, arthroscopic surgery and arthroplasty in terms of TMJ pain and mandibular mobility at a 60-month follow-up. ${ }^{12}$ The comparable therapeutic effects of different intervention modalities suggest that the least expensive, least invasive and simplest interventions should be employed for the initial management of TMD. ${ }^{11}$

Although promising outcomes have been reported with reversible treatment, the results remain controversial. $^{15}$ One prospective cohort study followed 40 TMD patients for 2.5 years and found that $43 \%$ of the patients were free of symptoms, $33 \%$ of the patients had decreased symptoms and $25 \%$ showed no improvement or required further treatment. ${ }^{15}$ Similarly, nearly $50 \%$ of patients who received reversible treatment reported no pain 5 years later, although $14 \%$ of patients continued to report significant pain. ${ }^{16}$ Little is known about the clinical characteristics of these TMD patients who experience a poor prognosis following reversible treatment. These findings suggest that prognostic information is critical when predicting the impact of reversible interventions in a population with TMD. However, there is a paucity of research that aims to identify prognostic factors associated with the clinical outcomes of reversible treatment in people with TMD. ${ }^{17-19}$ Previous studies have concluded that numerous biopsychosocial factors increase the risk of developing first-onset TMD; therefore, we speculated that these factors may have a theoretical association with prognosis in individuals with TMD. Moreover, the TMD status changes substantially over time, and acute TMD becomes chronic in $25 \%$ of patients, as the course of TMD progression can be highly variable. ${ }^{20}$ Long-term follow-up studies are needed to investigate the prognostic factors that influence the response to reversible treatment and the risk factors that are associated with the development of chronic TMD.

The aims of this prospective cohort study are: (1) to identify prognostic factors that are associated with clinical outcomes of reversible treatment in patients with TMD; and (2) to analyse risk factors that influence the development of chronic TMD during a 1-year follow-up period. Based on current knowledge of risk factors for the development of TMD that have been reported in epidemiological studies, putative prognostic factors, including demographic data, data from self-report questionnaires and measures of physical function will be collected and analysed.

\section{Specific aims}

Aim 1

To determine whether demographic characteristics and biopsychosocial factors are associated with the prognosis of reversible treatment for TMD.

Aim 2

To determine the risk factors associated with chronic TMD.

\section{TRIAL DESIGN AND METHODS}

This clinical-based, prospective, cohort study will be conducted at Shanghai Ninth People's Hospital, Shanghai Jiao Tong University School of Medicine. This protocol has been designed in accordance with the Standard Protocol Items: Recommendations for Interventional Trials (SPIRIT) 2013 statement and Prognosis Research Strategy (PROGRESS) guidelines. ${ }^{21}$ The results of this study will be reported in accordance with the Strengthening the Reporting of Observational Studies in Epidemiology (STROBE) statement. ${ }^{22}$

\section{Participants}

We will recruit 834 patients with TMD presenting to the rehabilitation department. Consecutive eligible patients will receive 1-4 weeks of reversible treatment and will be followed up for 12 months after baseline measurements. The reversible treatment programme has been reproduced from a previous systematic review on patients with TMD. ${ }^{23}$ The 1-year follow-up has been preregistered before patient enrolment. Study recruitment will commence in December 2020 and will be completed by December 2021.

The participants must meet the following inclusion criteria:

1. Patients aged $20-45$ years.

2. Patients fulfilling the Diagnostic Criteria for Temporomandibular Disorders (DC/TMD) ${ }^{24}$

3. Patients with myofascial pain or reducible or nonreducible disc displacement.

4. Patients should have one of the following symptoms: (A): visual analogue scale (VAS) score for orofacial pain $\geq 4$; (B): maximal mouth opening $<35 \mathrm{~mm}$.

5 . Understanding of the survey and ability to independently complete the questionnaires.

6. Patients must volunteer to participate in the study and sign the consent form. 
7. Patients receiving at least one type of reversible treatment (eg, education, self-management, medication, therapeutic exercise, manual therapy and occlusal splint therapy).

The participants will be excluded if they meet one of the following conditions at baseline:

1. History of traumatic facial injury or surgery.

2. Malignant disease, active rheumatic disease, haemorrhagic disease, heart disease or heart failure.

3. Pregnant or lactating women or women who plan to be pregnant within the next year.

Drop-out criteria: participants will have the right to drop out of the study at any time. Participants who meet one of the following conditions will be removed from the study:

1. The researcher believes that removal from the study will benefit the patient.

2. Failure to adhere to the follow-up time schedule or refusal to respond to the required questionnaires.

\section{Recruitment}

Patients will be recruited from the rehabilitation department of Shanghai Ninth People's Hospital, Shanghai Jiao Tong University School of Medicine. We will recruit eligible patients at their first clinic visit. Prior to study initiation, training will be delivered to the clinical examiners working at the physiotherapy clinic to inform them of the study and how to screen patients for eligibility. LX will serve as the reference examiner throughout the study. In a separate training session, each study examiner will conduct more than 10 blinded, replicated examinations of non-study volunteers. Data from the blinded, replicate examinations will be analysed for interexaminer reliability computed using the kappa statistic. Providers will have copies of the screening form to screen potential patients according to the inclusion and exclusion criteria. At the first outpatient visit, a potential patient will be informed about the study. All study participants will be required to provide written informed consent at the time of recruitment.

\section{Candidate prognostic factors}

Owing to the lack of consensus on the prognostic factors that influence the response to reversible treatment for TMD, demographic data, physical measurements, data from self-report questionnaires and information about the type of treatment modality will be collected. Putative factors have been selected based on current knowledge of risk factors for the development of TMD from epidemiological studies that may have a theoretical association with prognosis in individuals with TMD, as confirmed by the biopsychosocial model of developing TMD. These selected factors are feasible to measure in clinical settings. The candidate prognostic factors are summarised in table 1. All data collection will be standardised using clinical report forms and protocols.

Table 1 Summary of measures that will be collected

\begin{tabular}{|c|c|c|c|c|}
\hline Domain/candidate predictor & Baseline & Month 3 & Month 6 & Month 12 \\
\hline \multicolumn{5}{|l|}{ Fill out by clinicians } \\
\hline Inclusion or exclusion standard table & $\sqrt{ }$ & & & \\
\hline Sign informed notice & $\sqrt{ }$ & & & \\
\hline Demographic data and case history & $\sqrt{ }$ & & & \\
\hline Intercurrent diseases & $\sqrt{ }$ & & & \\
\hline Anterior maximal opening & $\sqrt{ }$ & $\sqrt{ }$ & $\sqrt{ }$ & $\sqrt{ }$ \\
\hline Head and neck posture & $\sqrt{ }$ & & & \\
\hline Adverse events & $\sqrt{ }$ & $\sqrt{ }$ & $\sqrt{ }$ & $\sqrt{ }$ \\
\hline Clinical routine inspection & $\sqrt{ }$ & & & \\
\hline $\mathrm{CPI}$ & & $\sqrt{ }$ & $\sqrt{ }$ & $\sqrt{ }$ \\
\hline \multicolumn{5}{|l|}{ Filled out by subjects } \\
\hline VAS score & $\sqrt{ }$ & $\sqrt{ }$ & $\sqrt{ }$ & $\sqrt{ }$ \\
\hline OBC & $\sqrt{ }$ & & & \\
\hline PHQ-15 & $\sqrt{ }$ & & & \\
\hline PHQ-9 & $\sqrt{ }$ & & & \\
\hline GAD-7 & $\sqrt{ }$ & & & \\
\hline PSQI & $\sqrt{ }$ & & & \\
\hline JFLS & $\sqrt{ }$ & $\sqrt{ }$ & $\sqrt{ }$ & $\sqrt{ }$ \\
\hline
\end{tabular}

CPI, characteristic pain intensity; GAD, Generalized Anxiety Scale; JFLS, Jaw Functional Limitation Scale; OBC, Oral

Behaviours Checklist; PHQ, Patient Health Questionnaire; PSQI, Pittsburgh Sleep Quality Index; VAS, visual analogue scale. 


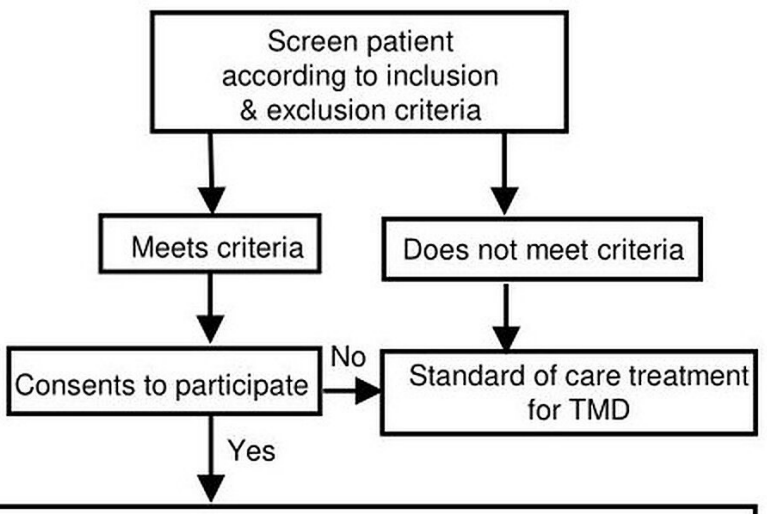

-Baseline Interview: demographic data, case history, intercurrent diseases, VAS

-Questionnaires: OBC, PHQ-15, PHQ-9, GAD-7, PSQI, JFLS

- Clinical examinations: TMJ sound, anterior maximal opening, head and neck posture

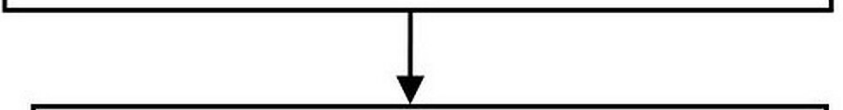

Reversible treatment: education, self-management, medication, physiotherapy, and splint therapy

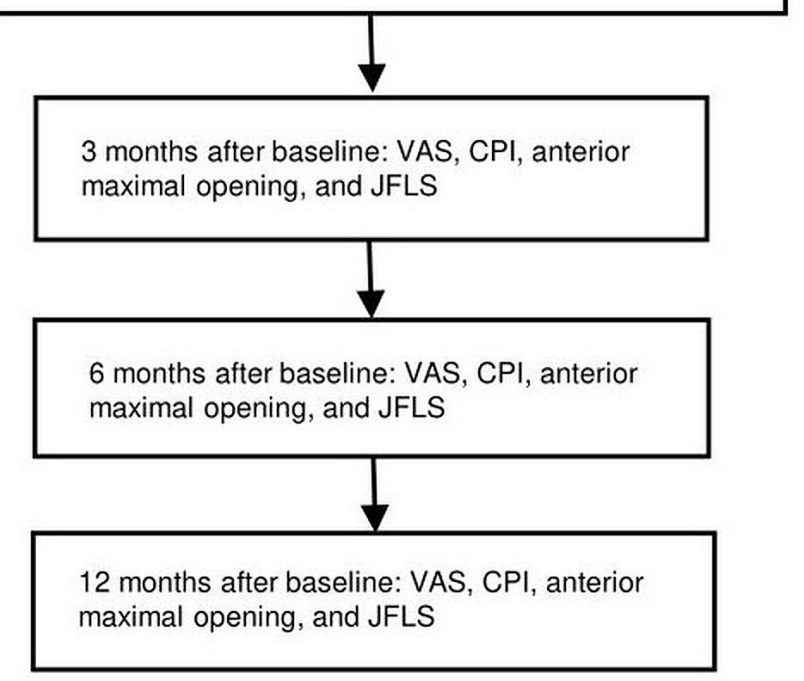

Figure 1 Participants recruitment and flow of the study. $\mathrm{CPI}$, characteristic pain intensity; GAD, generalised anxiety disorder; JFLS, Jaw Functional Limitation Scale; OBC, Oral Behaviours Checklist; $\mathrm{PHQ}$, Patient Health Questionnaire; PSQI, Pittsburgh Sleep Quality Index; TMD, temporomandibular disorders; VAS, visual analogue scale; TMJ, temporomandibular joint.

\section{Data collection}

Baseline data, including demographic data, data from self-report questionnaires and physical assessments, will be collected by a trained assessor at the first clinic visit. Patients will receive 1-4 weeks of reversible treatment within 2 weeks following recruitment, and the treatment modalities that patients receive will be recorded. Patients will be contacted by the same assessor by telephone 3, 6 and 12 months after baseline measurements to complete the physical examination and functional questionnaires (figure 1).

\section{Baseline interview, self-report questionnaires and physical examination}

During the first clinic visit, the researcher will collect each patient's demographic data (eg, sex, age distribution, educational attainment and marital status), case histories, intercurrent diseases (eg, pain related to the neck and TMJ region, and general musculoskeletal pain elsewhere) and use of medication. Anamnestic data will be collected at baseline, including the nature, duration and intensity of the pain. The intensity of the present facial pain, including masticatory muscle pain and TMJ pain at rest and during movements of the mandible according to the DC/TMD criteria, will be assessed with the VAS. The VAS scale is a measurement instrument that quantifies an attitude or characteristic that ranges across a continuum of values and cannot be directly measured. ${ }^{25}$ Operationally, it is usually depicted using a horizontal line, $100 \mathrm{~mm}$ in length, with word descriptors at each end. The patient marks the point on the line that represents their current feelings. The VAS score, measured in millimetres, is determined as the distance from the left end to the point that the patient marks.

Patients will be asked to complete self-report questionnaires and will follow the procedure shown in table 1 . Oral behaviours will be assessed at baseline using the Oral Behaviours Checklist, which is a self-report questionnaire comprising 21 items used to quantify the frequency of oral behaviours and has been evaluated as part of a larger study of the diagnostic validity and reliability of techniques for diagnosing TMD. ${ }^{26}$

The Patient Health Questionnaire (PHQ) is a selfadministered diagnostic instrument for common mental disorders. The PHQ-15 assessed 15 somatic symptoms from the PHQ, and the PHQ-9 comprises nine items to establish a depressive disorder diagnosis, in addition to grading depressive symptom severity. ${ }^{27}$ Generalised anxiety disorder (GAD) is a common mental disorder among TMD patients, and a seven-item anxiety scale (GAD-7) is a self-report scale to determine probable cases of GAD, with good reliability and procedural validity. ${ }^{28}$

The Pittsburgh Sleep Quality Index provides a valid, standardised, clinically useful measure of a variety of sleep disturbances that may affect sleep quality. ${ }^{29}$

The Jaw Functional Limitation Scale (JFLS) comprises 20 items used to assess limitations of jaw function in patients with TMD. ${ }^{30}$

Finally, a functional examination of the masticatory system and diagnosis of patients according to the DC/ TMD criteria ${ }^{24}$ will be conducted by the same clinician. Clinical stomatognathic assessments of TMD include the range of motion of the mandible, TMJ sounds and a patient's head and neck posture. ${ }^{31}$ The range of motion of the mandible will be measured with a Vernier calliper. ${ }^{25}$ When performing measurement of the anterior maximal opening, the examiner will ask the patient to place the 
mandible in a comfortable position. The patient will be asked to open the mouth as far as possible without assistance. The edge of the millimetre ruler will be placed at the incisal edge of the maxillary central incisor for maximal vertical orientation to the labio-incisal edge of the opposing mandibular incisor. This measurement will be considered the interincisal opening. If subjects open their mouth less than $30 \mathrm{~mm}$, the process will be repeated to ensure understanding. If the second opening is still less than $30 \mathrm{~mm}$, the measurement will be recorded as the interincisal opening. To measure the vertical incisal overlap, the patient will be asked to perform the action of biting to bring the teeth together. The line where the incisal edge of the same previously measured maxillary central incisor overlaps the mandibular incisor will be marked with a pen. The distance from the mandibular incisal edge to the marked line will be recorded as the vertical incisal overlap. The anterior maximal opening will be considered as the sum of the interincisal opening and the vertical incisal overlap. ${ }^{32}$

The objective method of assessing head and neck posture will be to measure the craniovertebral (CV) angle and the cranial rotation angle. ${ }^{33}$ The $\mathrm{CV}$ angle will be defined as the angle between the horizontal plane (the line perpendicular to true vertical axis) and the line extending from the tragus of the ear to the $\mathrm{C} 7$ spinous process. ${ }^{34}$ The cranial rotation angle will be formed by a line connecting the lateral canthus and the tragus with a horizontal line. ${ }^{34}$ Measurement of the cervical angle will be performed using a protractor. The digitisation procedure has been proven to be highly reliable. ${ }^{33}$ Sagittal plane imaging of the upper body of each patient will be conducted using a digital camera in a habitual relaxed, seated position. The patient will be asked to assume a comfortable habitual sitting position with the eyes focused toward the front, and the height of the chair will be $45 \mathrm{~cm}$. Red markers will be placed over the tragus and $\mathrm{C} 7$ spinous process by the same examiner. To ensure consistency in the images, the distance between the camera and the patient will be $1.5 \mathrm{~m}$, and the camera will be adjusted to remain aligned with the patient's shoulder.

\section{Interventions}

Patients included in the study will be invited to attend a study session at the physiotherapy clinic. At this session, patients will receive a standardised reversible treatment programme within 2 weeks of recruitment and will be followed up for a period of 12 months after baseline. According to the recommendations from the current systematic reviews and meta-analyses, the least invasive, simplest and reversible interventions are considered as first-line therapy options for TMD. An experienced physiotherapist will design a reversible treatment programme according to each patient's clinical symptoms, characteristics and willingness. The programme will include education, self-management, medication, therapeutic exercise, manual therapy and occlusal splint therapy, as previously reported. ${ }^{23}$ Patients will be informed of the reasoning behind the treatment plan and provided detailed information about it.

For patients who meet the inclusion criteria but refuse to participate in the study, the standard of care treatment will be given. To promote patient retention, the researcher will inform the potential patient that although they can withdraw at any time, dropping out without a reason reduces the ability to answer the research question and, therefore, weakens the study. Patients will be advised to carry on with their usual daily routines, and any interventions received during reversible therapy sessions will be recorded for a descriptive analysis. The details of the treatment (eg, time, number, duration and modality) and the number of and reasons for dropouts will be documented and reported, as well as any adverse events during the study. Participants will be monitored during the 1-4 week programme and the 12-month follow-up period. Data from patients receiving monotherapy or adjuvant therapy will be analysed separately.

\section{Outcomes}

The primary treatment outcome measures will be changes in the anterior maximal opening, worsening of TMD pain assessed using the VAS and changes in pain characteristics. A previous study concluded that the greatest improvement occurs between 3 and 4 months after baseline ${ }^{35}$; therefore, 3 months was chosen as the treatment outcome evaluation time point in this study. An anterior maximal opening of $\geq 35 \mathrm{~mm}$ and a reduction in VAS scores of at least $30 \% 3$ months after baseline will be defined as good clinical outcomes. ${ }^{17}$ Additional outcome measures will include changes in the frequency of TMD pain (recurrent, persistent and and one-time experience), JFLS scores and the diagnosis of chronic TMD. The patients will be asked to report discomfort and complications associated with the reversible treatment and how often they perform the treatment. Additionally, all outcome measures will be evaluated 6 and 12 months after baseline measurements and will be assessed using predictive modelling.

\section{Data management}

All data will be entered into the research folder, and a researcher will transfer them into the master data spreadsheet. Privacy of patient data will be maintained for all data handling procedures (collection transfer, storage and processing). The accuracy of the data will be guaranteed through a secondary review by study coauthors. Data recorded from each participant will anonymised using research numbers and will be accessible only by members of this research team. A spreadsheet will be stored on a portable drive, and the research folders will be locked in a cabinet.

\section{Trial organisation and monitoring}

The research team will consist of the authors listed in this article, in addition to administrative staff at the physiotherapy clinic who will assist with the entire process of 
the study and data entry. The primary investigator will manage the study flow and perform inspections of enrolment, treatment and procedures throughout the entire study. Other investigators will monitor data collection and facilitate the maintenance of data integrity through periodic evaluations during the data collection phase.

\section{Data analysis}

Numbers of individuals will be recorded, including those who are potentially eligible, confirmed eligible, recruited into the study, receiving reversible treatment and completing follow-up. Numbers related to withdrawals and loss to follow-up will be reported, along with reasons for removal from the study. Descriptive analyses of patients at baseline will include demographic, selfreport questionnaire and physical assessment data.

All analyses will be performed using Statistical Package for the Social Sciences (SPSS) software (V.25.0). The Kolmogorov-Smirnov test will be performed to assess whether the data are normally distributed $(p>0.05)$, considering that both parametric and non-parametric tests will be used in the data analyses. Linear regression, unpaired t-tests, $\chi^{2}$ tests and logistic regression will be used depending on the analysis to be performed. A multiple linear regression analysis will be conducted to develop a linear model to determine the associations between candidate prognostic factors and the response to reversible treatment in patients with TMD, with anterior maximal opening and TMD pain as continuous dependent variables. For assessing risk factors for the development of chronic TMD, univariate associations between categorical variables (treatment, sex, etc) and the diagnosis of chronic TMD according to the DC/TMD criteria will be evaluated using $\chi^{2}$ tests. Continuous variables (age, height, etc) and the diagnosis of chronic TMD according to the DC/TMD criteria will be evaluated using Student's unpaired t-tests with a Bonferroni correction. Multivariate analyses will be performed using binary logistic regression with forced entry of all independent variables. All analyses will be two tailed, with a threshold for statistical significance of $\mathrm{p}<0.05$.

\section{Sample size calculation}

This prospective cohort study was designed with a target sample size of 834 enrolled patients with TMD to investigate the association between 20 candidate prognostic factors and the clinical outcome of reversible treatment in patients with TMD over the 1-year follow-up period, assuming $20 \%$ loss to follow-up. The researchers will ensure that there are at least 10 participants per prognostic factor to conduct an adequately powered linear regression analysis. ${ }^{36}$ In a previous study, $70 \%$ of TMD patients who received reversible treatment reported good outcomes. ${ }^{12}$ Therefore, a sample size of 834 participants will be adequate to power a linear regression analysis of 20 candidate prognostic factors.

\section{Management of missing data}

For each variable of interest, the number of patients with missing data will be reported. Any potential bias due to loss to follow-up will be assessed and compared using baseline data of patients who withdraw or are lost to follow-up. ${ }^{37}$ Multiple imputation will be used to deal with missing outcome data, if necessary. ${ }^{38}$ Participants will be excluded from the predictive model and subsequent analyses if they request to withdraw from the study following recruitment. ${ }^{37}$

\section{Patient and public involvement}

No patients were involved with the design or will be involved in data collection, analysis or publication of the study.

\section{DISCUSSION}

This will be the first protocol to describe methods and analysis for identifying prognostic factors associated with clinical outcomes of reversible treatment in individuals with TMD. In particular, self-report measures combined with physical examinations will be incorporated to predict poor outcomes of reversible treatments in individuals with TMD. The candidate prognostic factors have been selected based on current knowledge of risk factors for developing first-onset TMD and their possible utilisation in clinical practice. The knowledge gained through this study will provide a better understanding of how these prognostic factors can be used to improve clinical outcomes, including whether reversible treatment is useful in the clinical management of TMD patients.

This study will be conducted in accordance with the SPIRIT statement and PROGRESS framework. ${ }^{21} 37$ The results of this study will provide new insights into who is likely to benefit from reversible treatment versus who is likely to develop chronic TMD. Between $57 \%$ and $71 \%$ of patients seeking treatment for acute TMD continue to report significant pain 6 months later. ${ }^{9}{ }^{39}$ Therefore, the evaluation of prognoses will be valuable for treatment planning for patients with TMD. In clinical practice, the heterogeneity of patient characteristics and psychosocial factors may be considered in treatment planning.

Despite the novelty of this trial, this study has some limitations. First, the candidate prognostic factors have been selected based on reported risk factors for developing first-onset TMD; however, some possible prognostic factors may be ignored. In future studies, we may include more candidate prognostic factors. Second, since the reversible treatment is a combined treatment, we cannot ascertain the effects of medication or manual therapy alone; however, we will explore the prognosis associated with each treatment component.

\section{ETHICS AND DISSEMINATION}

The protocol was approved by the Ethics Committee of Ninth People's Hospital, Shanghai Jiao Tong University School of Medicine, Shanghai, China, based on the guidelines set forth in the Declaration of Helsinki (SH9H2019-T316-4). An ethics review protects human medical 
research participants to ensure compliance with federal regulations. Any modifications to the protocol that may impact study procedures or the conduct of the study will require approval by the Institutional Review Board and a formal amendment to the protocol. This clinical trial has been registered with the Chinese Clinical Trial Registry (www.chictr.org.cn).

All study participants will provide written informed consent prior to randomisation. Patients included in this study have the right to withdraw at any time, and the reasons will be documented. If participants have trouble complying with the intervention or completing follow-up testing, they can discuss these challenges with the study coordinator. If participants miss measurement appointments, up to three reminders will be sent, and if necessary, the participant will be contacted by telephone to rearrange an appointment for measurements at an appropriate time. Regardless of the outcome, the results of the trial will be reported in accordance with the STROBE guidelines in a relevant scientific journal.

\section{TRIAL STATUS}

Recruitment started in December 2020 and is estimated to be completed in December 2021.

Contributors All authors are involved in the design of the study. LX initiated the study. BC, LX, SL, LZ and SF contributed to the planning and design. LZ, BC and LX drafted the study protocol and design. WS and SF will perform the statistical analysis. LX, LZ, YY and SF are responsible for managing the research. $L X$ is the supervisor of the project.

Funding This work is supported by the Clinical Research Program (Grant no. JYLJ201901) and the Shanghai Municipal Science and Technology Major Project (Grant No. 19441908400).

Competing interests None declared.

Patient and public involvement Patients and/or the public were not involved in the design, or conduct, or reporting, or dissemination plans of this research.

Patient consent for publication Not required.

Provenance and peer review Not commissioned; externally peer reviewed.

Open access This is an open access article distributed in accordance with the Creative Commons Attribution Non Commercial (CC BY-NC 4.0) license, which permits others to distribute, remix, adapt, build upon this work non-commercially, and license their derivative works on different terms, provided the original work is properly cited, appropriate credit is given, any changes made indicated, and the use is non-commercial. See: http://creativecommons.org/licenses/by-nc/4.0/.

ORCID iD

Bin Cai http://orcid.org/0000-0002-8573-9044

\section{REFERENCES}

1 Lipton JA, Ship JA, Larach-Robinson D, Duckro PN, Tait $\mathrm{RC}$, Margolis RB. Estimated prevalence and distribution of reported orofacial pain in the United States. J Am Dent Assoc 1993;124:115-21.

2 Sharma S, Wactawski-Wende J, LaMonte MJ, et al. Incident injury is strongly associated with subsequent incident temporomandibular disorder: results from the OPPERA study. Pain 2019;160:1551-61.

3 Wänman A, Marklund S. Treatment outcome of supervised exercise, home exercise and bite splint therapy, respectively, in patients with symptomatic disc displacement with reduction: a randomised clinical trial. J Oral Rehabil 2020;47:143-9.
4 Slade GD, Ohrbach R, Greenspan JD, et al. Painful temporomandibular disorder: decade of discovery from OPPERA studies. J Dent Res 2016;95:1084-92.

5 Ohrbach R, Bair E, Fillingim RB, et al. Clinical orofacial characteristics associated with risk of first-onset TMD: the OPPERA prospective cohort study. J Pain 2013;14:T33-50.

6 Sanders AE, Slade GD, Bair E, et al. General health status and incidence of first-onset temporomandibular disorder: the OPPERA prospective cohort study. J Pain 2013;14:T51-62.

7 Fillingim RB, Ohrbach R, Greenspan JD, et al. Associations of psychologic factors with multiple chronic overlapping pain conditions. J Oral Facial Pain Headache 2020;34:s85-100.

8 Greenspan JD, Slade GD, Rathnayaka N, et al. Experimental pain sensitivity in subjects with temporomandibular disorders and multiple other chronic pain conditions: the OPPERA prospective cohort study. J Oral Facial Pain Headache 2020;34:s43-56.

9 Fillingim RB, Slade GD, Greenspan JD, et al. Long-term changes in biopsychosocial characteristics related to temporomandibular disorder: findings from the OPPERA study. Pain 2018;159:2403-13.

10 Sanders AE, Greenspan JD, Fillingim RB, et al. Associations of sleep disturbance, atopy, and other health measures with chronic overlapping pain conditions. J Oral Facial Pain Headache 2020;34:s73-84.

11 Al-Baghdadi M, Durham J, Araujo-Soares V. TMJ disc displacement without reduction management: a systematic review. J Dental Res 2014;93:37S-51.

12 Schiffman EL, Velly AM, Look JO, et al. Effects of four treatment strategies for temporomandibular joint closed lock. Int J Oral Maxillofac Surg 2014;43:217-26.

13 Diraçoğlu D, Saral IB, Keklik B, et al. Arthrocentesis versus nonsurgical methods in the treatment of temporomandibular disc displacement without reduction. Oral Surg Oral Med Oral Pathol Oral Radiol Endod 2009;108:3-8.

14 Giannakopoulos NN, Rauer A-K, Hellmann D, et al. Comparison of device-supported sensorimotor training and splint intervention for myofascial temporomandibular disorder pain patients. J Oral Rehabil 2018;45:669-76.

15 Kurita K, Westesson PL, Yuasa H, et al. Natural course of untreated symptomatic temporomandibular joint disc displacement without reduction. J Dent Res 1998;77:361-5.

16 Ohrbach R, Dworkin SF. Five-Year outcomes in TMD: relationship of changes in pain to changes in physical and psychological variables. Pain 1998;74:315-26.

17 Grossi ML, Goldberg MB, Locker D, et al. Reduced neuropsychologic measures as predictors of treatment outcome in patients with temporomandibular disorders. J Orofac Pain 2001;15:329-39.

18 Burris JL, Evans DR, Carlson CR. Psychological correlates of medical comorbidities in patients with temporomandibular disorders. J Am Dent Assoc 2010;141:22-31.

19 Lipton JA, Marbach JJ. Predictors of treatment outcome in patients with myofascial pain-dysfunction syndrome and organic temporomandibular joint disorders. J Prosthet Dent 1984;51:387-93.

20 Rollman A, Visscher CM, Gorter RC, et al. Improvement in patients with a TMD-pain report. A 6-month follow-up study. J Oral Rehabil 2013;40:5-14.

21 Chan A-W, Tetzlaff JM, Altman DG, et al. Spirit 2013 statement: defining standard protocol items for clinical trials. Ann Intern Med 2013;158:200-7.

22 Elm V, Erik A, et al. The strengthening the reporting of observational studies in epidemiology (STROBE) statement: guidelines for reporting observational studies. Ann Intern Med 2007;156:568-75.

23 Gauer RL, Semidey MJ. Diagnosis and treatment of temporomandibular disorders. Am Family Phys 2015;91:378-86.

24 Türp G, Christoph J. Diagnostic criteria for temporomandibular disorders (DC/TMD) - presentation of the axis I classification. $J$ Craniomandibular Function 2014;23:45-51.

25 Katyayan PA, Katyayan MK, Shah RJ, et al. Efficacy of appliance therapy on temporomandibular disorder related facial pain and mandibular mobility: a randomized controlled study. J Indian Prosthodont Soc 2014;14:251-61.

26 van der Meulen MJ, Lobbezoo F, Aartman IHA, et al. Validity of the oral behaviours checklist: correlations between $\mathrm{OBC}$ scores and intensity of facial pain. J Oral Rehabil 2014;41:115-21.

27 Kroenke K, Spitzer RL, Williams JBW. The PHQ-15: validity of a new measure for evaluating the severity of somatic symptoms. Psychosom Med 2002;64:258-66.

28 Spitzer RL, Kroenke K, Williams JBW, et al. A brief measure for assessing generalized anxiety disorder: the GAD-7. Arch Intern Med 2006;166:1092. 
29 Buysse DJ, Reynolds CF, Monk TH, et al. The Pittsburgh sleep quality index: a new instrument for psychiatric practice and research. Psychiatry Res 1989;28:193-213.

30 Zhang Y, Chung SC, Chung JW. Evaluation of jaw functional limitation in TMD patients using a Korean version of jaw functional limitation scale (JFLS). J Oral Med Pain 2004;29:346-54.

31 Ohrbach R, Fillingim RB, Mulkey F, et al. Clinical findings and pain symptoms as potential risk factors for chronic TMD: descriptive data and empirically identified domains from the OPPERA case-control study. J Pain 2011;12:T27-45.

32 Dworkin SF, LeResche L. Research diagnostic criteria for temporomandibular disorders: review, criteria, examinations and specifications, critique. J Craniomandib Disord 1992;6:301-55.

33 Cheung Lau HM, Wing Chiu TT, Lam T-H, HMC L, Chiu TTW. Clinical measurement of craniovertebral angle by electronic head posture instrument: a test of reliability and validity. Man Ther 2009;14:363-8.
34 Joy TE, Tanuja S, Pillai RR, et al. Assessment of craniocervical posture in TMJ disorders using lateral radiographic view: a crosssectional study. Cranio 2019;9:1-7.

35 Pho Duc JM, Hüning SV, Grossi ML. Parallel randomized controlled clinical trial in patients with temporomandibular disorders treated with a CAD/CAM versus a conventional stabilization splint. Int $J$ Prosthodont 2016;29:340-50.

36 Royston P, Moons KGM, Altman DG, et al. Prognosis and prognostic research: developing a prognostic model. BMJ 2009;338:604:b604.

37 Riley RD, Hayden JA, Steyerberg EW, et al. Prognosis research strategy (progress) 2: prognostic factor research. PLoS Med 2013;10:e1001380-8.

38 Sterne JAC, White IR, Carlin JB, et al. Multiple imputation for missing data in epidemiological and clinical research: potential and pitfalls. BMJ 2009;338:2393:b2393.

39 Garofalo JP, Gatchel RJ, Wesley AL, et al. Predicting chronicity in acute temporomandibular joint disorders using the research diagnostic criteria. J Am Dent Assoc 1998;129:438-47. 